\title{
Mental Health Resources for At-Risk Adolescents
}

\author{
Amy Brackett, Nancy Duphily* \\ Department of Nursing, Fitchburg State University, USA \\ *Corresponding Author: Nduphily@fitchburgstate.edu
}

Copyright (C) 2013 Horizon Research Publishing All rights reserved.

\begin{abstract}
In considering the overall health of an individual, mental health plays an important role. Data from a search of the literature illustrate that for the number of children and adolescents experiencing mental health crises, resources are decidedly lacking, and many times, unavailable. The aim of this paper is to highlight the scarcity of mental health resources available for the at-risk adolescent, to address why these resources are crucial to access early on, and to discuss interventions to prevent, identify, and treat at-risk adolescents. Incorporated into this discussion is the impact of parents, peers, school, and home life as major contributors to the mental health of the adolescent.
\end{abstract}

Keywords Mental health resources, at risk adolescent, ADHD, depression, suicide, bipolar disorder

\section{Introduction}

For the first time in over 200 years, the life expectancy of a generation is projected to be shorter than that of the previous one [6].Mental health conditions facing children and adolescents today include depression, suicide, bipolar disorder and Attention Deficit Hyperactivity Disorder (ADHD). Studies show that 1 in 10 adolescents experience a mental health crisis that impairs their daily functioning. Of those adolescents with mental health problems, $80 \%$ do not receive appropriate mental health evaluations or services[6].Unfortunately, the resources available for adolescents experiencing a mental health crisis are severely lacking [4].

Almost $35 \%$ of all children in the United States do not see a doctor in a given year; those who did see a doctor may not have received a mental health evaluation, as it is not typically required in an annual physical examination [6]. According to the National Institute of Mental Health, only $6 \%$ of children did receive mental health services; of this population, $20 \%$ have a diagnosable mental health condition [1]. These statistics show that for the number of children and adolescents experiencing mental health crises, the resources are lacking, and many times, unavailable.
Although there are effective treatments for adolescents with mental health disorders, there are long delays (sometimes decades) between the first onset of symptoms and when adolescents receive treatment [9]. It is crucial to identify and treat mental health conditions early on in children and adolescents. In order to do so, more resources need to be available to this vulnerable population. Those resources include specialists in mental health, in hospitals, schools and clinics. Even more important is the resource of mental health education for adolescents and parents.

The aim of this paper is to highlight the scarcity of mental health resources available for the at-risk adolescent, to address why these resources are crucial to access early on, and to discuss interventions to prevent, identify, and treat at-risk adolescents. Incorporated into this discussion is the impact of parents, peers, school, and home life as major contributors to the mental health of the adolescent.

By addressing mental health issues facing adolescents in society today, it is the intent of this discussion to identify barriers to the treatment of adolescents in mental health crisis, and to describe health promotion strategies and resources that may be implemented in the care of this vulnerable population. Five mental health disorders experienced by the at-risk adolescent will be discussed.

Along with an extensive literature search, a local perspective was gained from a local high school Adjustment Counselor, who is both a registered nurse (BSN) and Licensed Independent Counselor Social Worker (LICSW). In a recent interview, the Adjustment Counselor gave her opinions of adolescent mental health based on her experience working with adolescents. She explained that in order to gather useful information, the guidance department will first talk to a student who may be experiencing a crisis. Following this initial step, a crisis assessment is performed, which includes a more in-depth interview with the student at risk to determine if intervention is needed. If a crisis is recognized, further evaluation and referral to a hospital or agency is the next step.

The Adjustment Counselor believes that there is a true scarcity of mental health services for the at-risk adolescent. Resources is crucial. In actuality, these services are the first to be eliminated, fallout from cost containment measures in 
organizations. Contributing to this problem is the fact that many health care professionals, already over-burdened in their practices, are not taking on new clients.

The reality exists that there are just not enough skilled professionals and practitioners accessible to meet the needs of this vulnerable population of adolescents. Further complicating this situation is the fact that finances present a barrier for families seeking treatment for their child. Too often, families cannot afford their co-pays, blocking access to care.

In the areas of diagnosis and treatment, the Adjustment Counselor believes that there are many misdiagnoses of ADHD and an overuse of drugs. When it comes to depression, she feels that careful and continuous monitoring with medication administration, along with and cognitive behavioral therapy, are most effective.

\section{Depression}

Adolescent depression severely impairs daily functioning such as concentration and thought processes and often recurs or persists into adulthood. Depression affects up to $20 \%$ of adolescents and as many as 1 in 5 teens experience major depression at one point. Symptoms of depression can range from irritable moods to fatigue and can often be mistaken for typical adolescent moodiness [6]. Because of this, depression can often be overlooked by parents or peers.

Factors that act as precursors to depression include parenting style, stressful life events, a major illness, academic struggles, family turmoil, low self- esteem and anxiety. It is important to address adolescent depression early, as it is a major contributor to adolescent suicide. Additionally, adolescents with a diagnosis of depression are at a higher risk for substance abuse. Studies have shown that substance abuse occurs concurrently with depression approximately $25 \%$ of the time [5].

Strategies for prevention include education in schools and at home, as well as in-depth screening adolescents for depression. Screening may be carried out by primary care physicians and should include key questions about mood or the inability to have fun. Risk factors for depression include a family history, low self-esteem, anxiety, academic struggles, family turmoil, or negative life events. After risk factors and screenings are taken into consideration, the doctor identifies a diagnosis based on a series of symptoms present in the same two week period [2].

When considering risks and benefits of treatment, one must also keep in mind the dangers of non-treatment. This includes an increased risk for substance abuse, conduct disorder, impaired functioning, and suicidal thoughts [2]. Additionally, alterations in the neurotransmission of norepinephrine and dopamine, and decreased levels of serotonin in the brain have been known to cause symptoms of depression [5].

An option in the treatment of depression involves the use of antidepressants. These medications work by inhibiting the reuptake of serotonin in the brain. There are a variety of different antidepressants, all with their own side effects. Any medication has risks, but there is evidence that antidepressants decrease the overall suicide rate when taken as directed, and when carefully monitored by the practitioner. One research study showed that each one-percent increase in antidepressant use was associated with a decrease of 0.23 suicides per 100,000 adolescents per year [2].

Another form of treatment for depression is Cognitive Behavioral Therapy (CBT), named for the several psychotherapeutic strategies used for depression. The framework for CBT is that thoughts, feelings, and behavior are all related. Treatment is aimed at teaching psychosocial and relaxation skills as well as analysis of cognition and behavior. The psychotherapeutic treatments shown to be most effective for adolescents with depression are CBT and antidepressant therapy [1,2].

\section{Suicide}

If adolescent depression is left untreated, it can spiral downward into suicidal thoughts. Suicide is often determined to be the result of a major depressive episode. In many cases this major depressive episode is the first one that the adolescent who commits suicide has experienced [6]. While terrifying to consider, it illustrates the importance of early recognition. Suicide is the third leading cause of death in adolescents in the United States. A reported one in five high school students have suicidal thoughts in a given year; one in ten have attempted suicide. Adolescent males account for alarming $84 \%$ of suicides for people ages 11-21 [2].

A suicidal assessment given to those at-risk adolescents involves questions about stressors, intent, plan, intensity of suicidal thoughts, and risk factors. Risk factors reported to contribute to suicidal behavior include the following: presence of psychiatric illness, with depression being most common, previous history of suicide attempts, low family and peer support, physical and sexual abuse, victimization, serotonin deficiency, having a family member who had attempted suicide, and access to firearms [8].After the assessment is completed and all factors taken into consideration, inpatient or outpatient treatment may be recommended.

The first step in preventing adolescent suicide is to increase awareness of the problem. A second measure promotes health care professionals' work with adolescents and the community to educate the public regarding mental health [8]. One such strategy for prevention is a "resource card". The resource card fits into a pocket or wallet and provides easy access 24 -hour crisis telephone numbers. This card comes with the additional benefit of discounts at local restaurants. For community members, area wide educational forums raise awareness of risk factors for adolescent suicide. Education can happen anywhere. Elementary and secondary schools, local colleges, recreation centers, hospitals and 
health providers' offices are but a few examples where free access to understanding and help can take place.

\section{Bipolar Disorder}

Bipolar disorder is characterized by episodes of both depressed and elevated mood, although the predominant experience is a low mood [7]. The peak age of onset is adolescence and early adulthood. Studies show that $20 \%$ of adults with bipolar disorder have experienced initial symptoms before the age of nineteen. An alarming $1 \%$ of adolescents are affected by bipolar disorder, who tend to suffer from longer episodes than those with a later onset. The major problem with bipolar disorder is that it is relatively common, yet goes unrecognized, misdiagnosed, and untreated [7].

In order for an adolescent to be diagnosed, the symptoms of mania, euphoria and irritability must be present at some point in time. Since individuals with bipolar disorder experience more depressive episodes than manic, they are at risk for suicide. Thorough evaluation is necessary and a plan of care should be implemented. The plan of care should identify any personal, social, or environmental risk factors, along with steps to be taken to access help in a crisis [7]. A mood diary is also recommended in which adolescents can write in a mood diary about how they are feeling that day. Diaries can be read by physicians or parents to recognize any mood patterns or determine causes of depressive moods.

Antipsychotic medications and mood stabilizers are the most common and more effective treatment of bipolar disorder in children and adolescents [7]. These medications can cause weight gain, which could present further problems for adolescents struggling with self-esteem or body image issues. Adolescents taking medication for bipolar disorder should monitor their weight and engage in health promotion activities such as a healthy diet and exercise routine.

Included in the treatment plan is cognitive behavioral therapy (CBT).As discussed previously in this paper in the treatment of depression, this intervention focuses on depressive symptoms of the bipolar disorder, problem solving, social functioning, guidance about lifestyle choices, and the need to take medication as prescribed. Exercise is also encouraged to cope with mild depressive symptoms in adolescents.

\section{Attention Deficit Hyperactivity Disorder (ADHD)}

Attention deficit hyperactivity disorder (ADHD) is the most commonly diagnosed mental health condition. Symptoms include difficulty paying attention and poor impulse control. ADHD can lead to poor academic performance, underachievement, aggression, and decreased social skills [6]. ADHD is the most common comorbid condition with bipolar disorder. Both ADHD and bipolar share common diagnostic characteristics such as distractibility, restlessness, and over talking; yet bipolar disorder is episodic, whereas ADHD is not [7]. Approximately two thirds of children and adolescents with ADHD have a comorbidity such as oppositional defiant disorder, depression, an anxiety disorder, conduct disorder, or learning disabilities [5]. Because of this comorbidity, it is extremely important to properly diagnose and treat ADHD.

Although the exact cause of ADHD is unknown, it is thought to be due to an alteration in the dopamine and norepinephrine neurotransmitter system in the brain. Diagnosis is based on the presence of six or more of the following symptoms: failure to pay attention, listen, follow through or complete tasks, and comprehend instructions. Symptoms may also include poor organization, careless mistakes on homework, losing things essential for completion of tasks, distractibility and forgetfulness [5].

When it comes to gender differences, ADHD has been shown to be more common in boys than girls by a ratio of $3: 1$ [5]. To identify and diagnose ADHD, the assessment should include a health history and physical examination. The history includes family history, age of onset of symptoms, how frequent symptoms are experienced, and any stressors that may cause symptoms. The physical examination includes height, weight, blood pressure, hearing and vision screening, behavior analysis and physical appearance examination

The best way to diagnose ADHD in adolescents is through standardized questionnaires, interviews with parents, adolescent and teachers, as well as personal observation. If ADHD symptoms are observed, a treatment plan is implemented. Stimulant medications are the most effective drug therapy used to treat ADHD today, and the medication Ritalin is the most widely prescribed. Eighty percent of adolescents respond to a stimulant medication.

In terms of duration of therapy, there are no recommendations as to how long an adolescent should remain on an ADHD medication, but it is not unusual for them to remain on a stimulant medication for several years with proper follow-up. A period off of a medication, known as a drug-holiday may be recommended as the adolescent enters teen years. This period should be during the school year to see if academics and social functioning are affected. If academics or social functioning is affected, medication may be continued. Studies have shown no long-term detrimental effects of stimulant use in adolescents

It is important to identify any comorbid conditions such as depression or anxiety before starting stimulant therapy for ADHD. Starting a stimulant medication with these conditions being untreated may exacerbate the comorbid conditions. Common side effects of stimulant medications are anorexia, weight loss, insomnia, headache, abdominal pain, or nervousness Alternative treatments for ADHD are nonpharmacological treatments such as counseling, cognitive behavioral therapy, or support groups.

ADHD is associated with comorbid conditions such as depression, anxiety, learning disorders, and bipolar disorder. Children and adolescents with ADHD are more likely to 
have low self-esteem, academic failure, and social difficulties; untreated, ADHD can lead to underachievement and aggression [8]. A major concern is that schools are being blamed for medicating their issues away by having students screened for and diagnosed with ADHD [8]. This concern can be connected to the scarcity of resources for the at-risk adolescent because schools are not accessing proper resources for their students.

Parents who have a child with ADHD may doubt their parenting abilities due to their child's high level of activity and inability to pay attention [7]. It is important for the family to be educated about ADHD and to learn behavioral techniques that work best such as time-out and positive reinforcement. Families may also be referred to local support groups.

\section{Connecting Newtown and Identifying the Scarcity of Resources}

In response to the Newtown shooting, the National Rifle Association argued that our lack of a national database of the mentally ill was the "real" problem, not access to guns[3]. This begs the question of whether we should shift the focus from gun control to who has the gun. Since some mental health disorders can lead to aggression and violence, this ultimately means that more access to mental health care is needed in order to reduce the risk of violence in adolescents. The current shortage of psychiatric hospital beds for children and adolescents in crisis further complicates the issue of getting timely and appropriate treatment [9].

Weknow that gaining access to mental health care is severely limited [3].A key issue raised by both the literature review and the Adjustment counselor interview concerns the elimination of programs crucial to adolescent mental health. As states slash their budgets, our mental health system lacks the capacity to care for those who need it most and sends many of the mentally ill into the criminal justice system $[3,9]$.

\section{Discussion}

One possible solution to identify mental health disorders early on in adolescents is to incorporate mental health checkups in primary care visits. The National Comorbidity Survey Replication Study shows that $50 \%$ of all mental health disorders present by age 14 [4]. Early signs of mental health disorders are often overlooked in adolescents for one reason or another, although, the average diagnosis occurs at least ten years after onset of symptoms [5]. Incorporating mental health screenings in primary care visits is a form of prevention and early detection.

According to the American Academy of Pediatrics (AAP), a mental health screening involves a two stage process [5]. The first stage is a scientifically tested questionnaire that can be completed by the adolescent in the waiting room and scored by a nurse or other medical professional. It may also be useful to have a parent complete a series of questions about their adolescent as well. The second phase of the screening includes the physician discussing the results of the questionnaire with the adolescent in order to determine what is causing the reported symptoms and if symptoms interfere with daily life. For adolescents whose symptoms interfere with their daily functioning, the physician may provide referral information to adolescent and parents [4].

\section{Nursing Implications}

Mental health conditions manifested at an early age that are overlooked, undetected or ignored place the growing adolescent at risk for dangerous behaviors. These behaviors include, but are not limited to, the reckless use of firearms, unsafe driving, substance abuse and self- harm. Because nurses are the ones closest to the client, they have a unique opportunity and ability to positively and significantly impact the lives of the individuals and families for whom they care. As caregivers, nurses' strengths lie in their ability to assess the client. As teachers, nurses arm themselves with current information and resources with which to develop prevention and treatment services. As managers, nurses are in a unique position to improve access, assessment and recognition of mental health issues for all clients.

Nurses are faced with mental health issues in every area of nursing practice: emergency departments, maternal child health, community agencies and intensive care units. Because of the exposure to mental health issues in the diverse areas of nursing practice, it is imperative that all nurses arm themselves with knowledge in best practices for caring with this vulnerable population. In turn, veteran nurses must "pay it forward" by sharing their education, experience and expertise, to mentor and empower the next generation of nurses.

Maintaining and encouraging other nurses to adopt an evidence-based practice philosophy must be a major part of this professional legacy. Additionally, nurses must become agents of change with by involving themselves politically, a challenge in the current scarcity of funding for mental health services. They must share their experiences and the lessons learned with those in administrations and above. Empowering clients and families to share their stories as well reinforces the message. The stories cannot be told only once, however; they must be repeated until they are heard. The awareness created from the voices of a few is a significant first step to making a positive change in the mental health of our at-risk youth.

\section{REFERENCES}

[1] Belfer, M. (2008). Child and adolescent mental disorders: the magnitude of the problem across the globe. Journal of Child Psychology \& Psychiatry, 49(3), 226-236. 
[2] Cook, M., Peterson, J., \& Sheldon, C. (2009). Adolescent depression: an update and guide to clinical decision making. Psychiatry (1550-5952), 6(9), 17-31.

[3] Gardner, D. B. (2013). Madness in America: Holding a Rational Conversation. Nursing Economic\$, 31(1), 50-51.

[4] Jackson Allen, P.L. (2011). Incorporating Mental Health Checkups into Adolescent Primary Care Visits. (2011). Pediatric Nursing, 37(3), 137-140.

[5] Kyle, Terri. (2008). Essentials of Pediatric Nursing. Lippincott Williams \& Wilkins, Philadelphia, PA.

[6] Manning, A. (2009). Bridging the gap from availability to accessibility: providing health and mental health services in schools. Journal of Evidence-Based Social Work, 6(1), 40-57.
[7] McDougall, T. (2009). Nursing children and adolescents with bipolar disorder: assessment, diagnosis, treatment, and management. Journal of Child \& Adolescent Psychiatric Nursing, 22(1), 33-39.

[8] Pirruccello, L. (2010). Preventing adolescent suicide: a community takes action. Journal of Psychosocial Nursing \& Mental Health Services, 48(5), 34-41.

[9] Rollins, J. A. (2013). The Aftermath of December 14, 2012. Pediatric Nursing, 39(1), 10-11.

[10] Woodard, R. (2006). Primary care approaches. The diagnosis and medical treatment of ADHD in children and adolescents in primary care: a practical guide. Pediatric Nursing, 32(4), 363-370. 